\title{
Morphologic analysis of the proximal tibia after open wedge high tibial osteotomy for proper plate fitting
}

\author{
Oui Sik Yoo ${ }^{1,2}$, Yong Seuk Lee ${ }^{3 *}$, Myung Chul Lee ${ }^{4}$, Jae Hong Park ${ }^{3}$, Jae Won Kim ${ }^{1}$ and Doo Hoon Sun ${ }^{1}$
}

\begin{abstract}
Background: After open wedge high tibial osteotomy (OWHTO), the proximal fragment resembles the anatomy of the proximal tibia that is aligned in the anterior-posterior direction and the distal fragment resembles the anatomy of the mid shaft that is aligned in the proximal-distal direction. In addition, the medial portion of the proximal fragment becomes aligned medially and the medial portion of the distal fragment, laterally, depending on the magnitude of the posterior opening gap. Therefore, there would be a mismatch between the post-correction bony surface and the previous pre-contoured plate geometry. The purpose of this study was to devise a new plate that best fit the post-contoured anatomy of the tibia by evaluating the surface geometry of the plate positioning site after OWHTO.
\end{abstract}

Methods: Thirty-one uni-planar and 38 bi-planar osteotomies were evaluated. Surgical indications were age of under 70 years, relatively active patient who performs recreational sports activities. Other indications were similar with general recommendation of HTO. Computed tomography (CT) of the operated knees was performed and it was used for the reconstruction of the 3D model. Bone model axis re-alignment was performed with coronal, sagittal, and axial plane. Morphologic analysis of the proximal tibia was performed using the following parameters: (1) radii in axial plane, 2) radii in coronal plane, and 3) angle and horizontal distance (Distance $X$ ) between the proximal and distal fragments. These were also analyzed according to the correction degree. The Analysis of Variance (ANOVA) test was conducted to verify the change depending on the correction amount of the posterior opening gap. The values obtained for the uniand bi-planar osteotomy were compared by the independent $t$-test.

Results: There were 9 male and 60 female patients were recruited to this study; the mean age was $58.3 \pm 8$ and $56.9 \pm 7$. 6 years, respectively. Preoperative weight bearing line (WBL) was $21.59 \pm 11.36$ and $22.32 \pm 10.55 \%$, respectively. Mean correction degree was $10.9 \pm 2.7$ and $11.1 \pm 2.6 \mathrm{~mm}$, respectively. The radii of the tibial cross-sectional contour at the head portion tended to increase from the proximal to distal direction. The radii of the tibial cross-sectional contour at the neck portion tended to decrease from the proximal to distal direction. The radii of the coronal plane tended to increase from the proximal to distal direction. The angle between the proximal fragment and the distal one varied with the correction amount of the posterior opening gap. Shaft_Mid and Distance X of Groupl (110.08 mm and $6.11 \mathrm{~mm}$, respectively) which had lower correction angle were lower than those of Groupll (130.05 mm and $6.41 \mathrm{~mm}$, respectively) and those of Grouplll (136.35 mm, $8.01 \mathrm{~mm}$, respectively) in coronal plane. There were significant differences ( $p=0.023$ $<0.05$ and $p=0.009<0.01$, respectively).

(Continued on next page)

\footnotetext{
* Correspondence: smcos1@hanmail.net

${ }^{3}$ Department of Orthopaedic Surgery, Seoul National University College of

Medicine, Bundang Hospital, 166 Gumi-ro, Bundang-gu, Seongnam-si,

Gyeonggi-do 463-707, South Korea

Full list of author information is available at the end of the article
} 
(Continued from previous page)

Conclusion: Current plate design should be modified to the surface geometry of the post-correction for the proper fitting. As the correction degree increases, the plate should be bended at the both end of the opening gap in coronal plane.

Trial registration: 'retrospectively registered (ISRCTN97792440).

Keywords: Knee, High tibial osteotomy, Stability, Plate, Fitting

\section{Background}

Open wedge high tibial osteotomy (OWHTO) is a wellestablished treatment for medial uni-compartmental arthritis of the knee joint [1-5]. To ensure proper load re-distribution, the osteotomy site should be opened at the medial side and the magnitude of the opening should be based on the preoperative deformity. After the correction, proper load re-distribution should be maintained during the healing process for improving the radiological and clinical outcomes. Therefore, stable fixation is needed for the safe healing of this additive type of osteotomy in order to minimize the risk of non-union and loss of correction [6].

A position HTO plate (such as a Puddu plate) is a short plate comprising an integrated spacer block, available in different sizes, corresponding to the amount of the medial opening desired [6]. However, a high platerelated complication rate and significant loss of correction have been being reported [7-10]. Recently, a long and rigid titanium plate (Tomofix, Depuy Synthes, PA, USA), which is anatomically pre-contoured to the medial tibial metaphysis, was designed specifically for OWHTO. This implant is equipped with locking bolts and, thus, functions as an internal plate fixator $[6,11,12]$. Nowadays, new anatomical locking metal block plates are available; it is also found that locking plates with an additional metal block are more stable than those without a metal block [2].

However, these plates are designed as a pre-contoured anatomy of the medial aspect of the proximal tibia. The proximal tibia has a unique 3-dimensional anatomy compared to the mid or distal tibia, because multiple structures including the tibia plateau, central fovea, and posterior cortex undergo changes abruptly [13-15]. After OWHTO, the proximal fragment resembles the anatomy of the proximal tibia that is aligned in the anterior-posterior direction and the distal fragment resembles the anatomy of the mid shaft that is aligned in the proximal-distal direction. In addition, the medial portion of the proximal fragment becomes aligned medially and the medial portion of the distal fragment, laterally, depending on the magnitude of the posterior opening gap (Fig. 1). Therefore, there would be a mismatch between the post-correction bony surface and the previous pre-contoured plate geometry.
The purpose of this study was to devise a new plate that best fit the post-contoured anatomy of the tibia by evaluating the surface geometry of the plate positioning site after OWHTO. The hypotheses tested in this study were as follows: (1) the anatomical plate should have an abrupt bending 3 dimensionally around the posterior opening gap and 2) the importance of this tendency increases with the increase in the posterior opening gap.

\section{Methods \\ Demographics}

From March 2012 to June 2014, 31 uni-planar and 38 biplanar osteotomies were retrospectively evaluated. All patients underwent OWHTO for treatment of medial unicompartmental osteoarthritis with a varus deformity. There were 9 male and 60 female patients were recruited to this study; the mean age was $58.3 \pm 8$ and $56.9 \pm$ 7.6 years, respectively. The mean body mass index (BMI) was $26.2 \pm 2.7$ and $27.9 \pm 3.9$, respectively. The mean range of motion (ROM) was $132 \pm 9.5$ and $133 \pm 11^{\circ}$, respectively. Preoperative weight bearing line (WBL) was $21.59 \pm$ 11.36 and $22.32 \pm 10.55 \%$, respectively, and mean correction degree was $10.9 \pm 2.7$ and $11.1 \pm 2.6 \mathrm{~mm}$, respectively. Preoperative demographic data of 2 groups were not statistically different (n.s.). The surgical indications were relatively active patients aged under 70 years and a varus arthritic knee. Additionally, per the general recommendations of HTO, some other conditions such as mild patellofemoral arthritis were also considered. The exclusion criteria were 1) OWHTO performed by cosmetic problem, 2) OWHTO performed by combined ligament instability, and 3) double osteotomy.

Computed tomography (CT) of the operated knees of the uni-planar and bi-planar group was performed at 2.5 days (range, 2-5 days), using CT scanners (SOMATOM Definition, Siemens, Forscheim, Germany; MX8000, Brilliance 64, Brilliance iCT, Philips, Netherland). The CT was conducted under the following conditions: tube voltage $(120 \mathrm{kV})$, tube current (250 mAs), pitch (0.609), slice thickness $(3 \mathrm{~mm})$, and resolution (standard). A continuous scan was obtained from approximately $10 \mathrm{~cm}$ above to $10 \mathrm{~cm}$ below the joint line. Institutional review board approval was obtained prior to initiation of the study, and all patients provided informed consent for participation. 


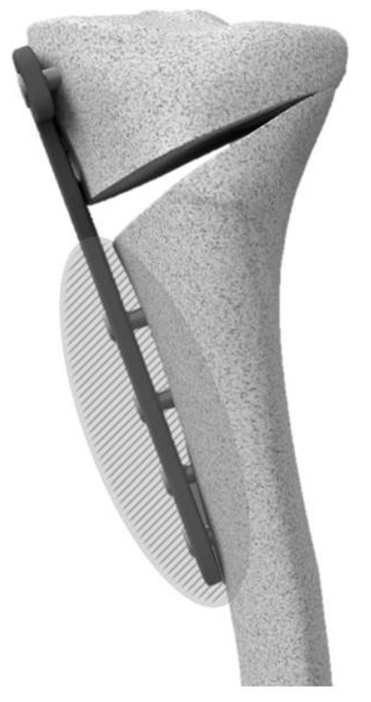

(A) Anterior View
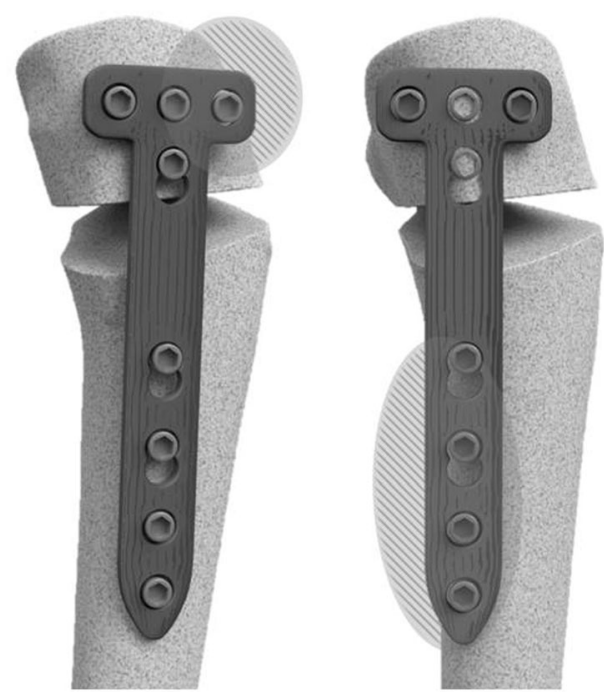

(B) Medial View

Fig. 1 Mismatch of the OWHTO plate on the osteotomized bony surface: Mismatch of the coronal plane occurs by moving distal fragment laterally $\mathbf{a}$ and mismatch of the sagittal plane occurs by different centers between the proximal and distal fragment $\mathbf{b}$

\section{Surgical technique}

An approximately $5 \mathrm{~cm}$ incision is made longitudinally at the $1 \mathrm{~cm}$ anterior portion of the posterior crest of the tibia. This incision is more posterior than the usual incision because it allows for easy insertion of a releaser and a protector. The interval behind the patellar tendon is now freed, and the insertion area of the tendon is protected using a retractor. Then, the superior border of the pes anserinus is incised, the medial collateral ligament is mobilized from the tibia, and release is performed by insertion of a periosteal elevator.

Release behind the posteromedial cortex of the tibia is typically done using gauge packing, which; enables access to the more than half of the posterior cortex of the tibia. After removal of the gauge, the releaser is inserted through this interval and further release is performed by pushing the releaser until making contact is made with the posterior cortex. If the tip of the releaser reaches to the fibular head area, the protector is inserted at the interval between the posterior cortex and the releaser, and the releaser is removed.

With the help of anterior-posterior C-arm image, the tip of the protector is hooked to the target portion of the hinge located at the lateral cortex of the proximal tibia. Then, the cutting block is attached to the protector and pushed to the posteromedial cortex of the tibia. If contact is made, the cutting block is tightened to the protector and guiding pins are inserted at the four holes of the cutting block [16]. Finally, sawing is performed; the main goal of this procedure is sawing of the posterior cortex. After removal of the protective cutting complex,
C-arm images are checked. Finally, in the bi-planar osteotomy, anterior retrotubercle osteotomy is performed, with distraction at the most posterior gap. The amount of distraction or control of tibial slope is adjusted according to pre-operative planning and it was adjusted at the most posterior gap [17]. Usually, target point was around $62.5 \%$ of the weight bearing line (WBL) and was adjusted from 55 to $65 \%$ according to the status of the medial compartment. If the medial compartment showed severe degeneration, it was shifted to the larger correction of approximately $65 \%$.

\section{Evaluations}

Postoperative CT data obtained after OWHTO using a locking plate were used for the reconstruction of the 3D model with Mimics v.16.0 (Materialise, Leuven, Belgium) of the proximal tibia and locking plate. Segmentation of the region of interest (ROI) was obtained from Digital Image Communication in Medicine (DICOM) data, and ROI was determined by adjusting and setting of Hounsfield Units (HU) threshold. Then, reconstruction was performed from the segmentation by stacking process of the contour [18, 19] (Fig. 2).

Axis re-alignment was needed during reconstruction of the 3D model because the coordinates of the postoperative $\mathrm{CT}$ data were respectively different according to the posture of the patient and their progress after surgery. The coronal axis was re-aligned with the distal tibia center to the tibial spine, the sagittal axis was re-aligned in the same line with the fibula, and the axial (transverse) axis was re-aligned with the center of posterior 


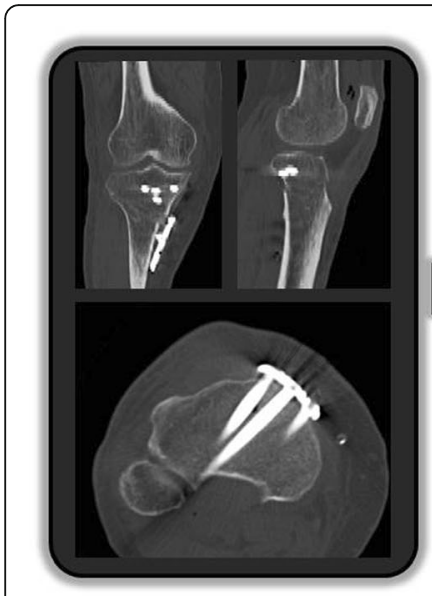

(A)

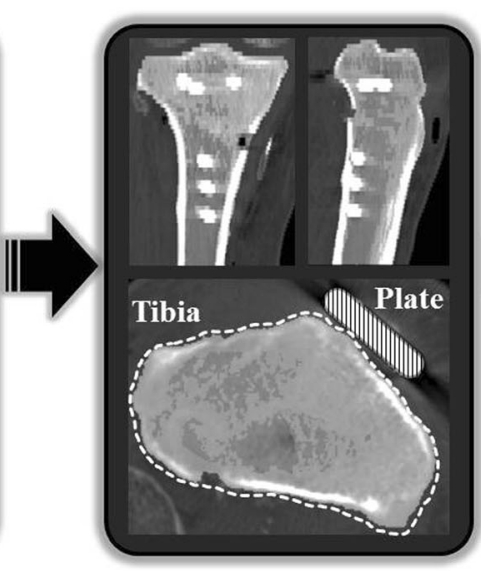

(B)

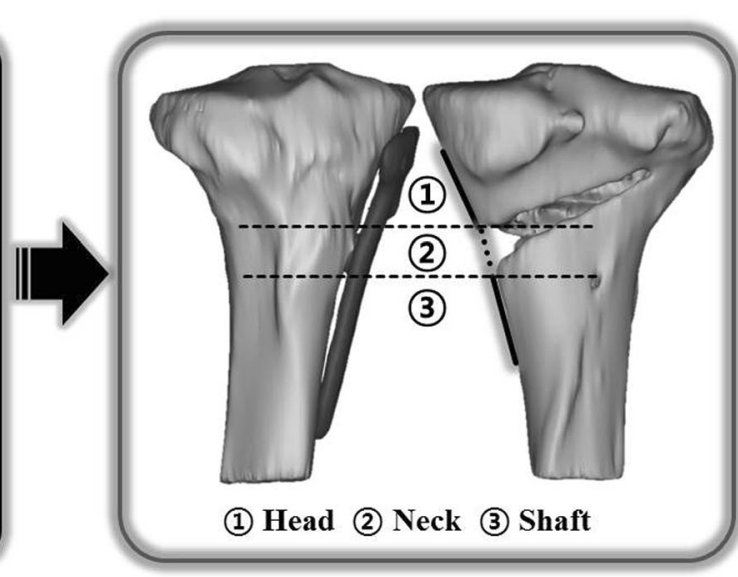

(C)

Fig. 2 Modeling is performed by acquisition of the data a selection of the $\mathrm{ROI} \mathbf{b}$ and $3 \mathrm{D}$ reconstruction process $\mathbf{c}$

cruciate ligament (PCL) insertion to the medial onethird of the tibial tuberosity using SolidWorks 2014 (Dassault Systemes, Waltham, Massachusetts, USA) (Fig. 3). Morphologic analysis of the proximal tibia was performed using the following parameters (Y.O.S. and K.J.W., experience of more than 5 years): (1) radii in axial plane, 2) radii in coronal plane, and 3) angle and horizontal distance (Distance $\mathrm{X}$ ) between the proximal and distal fragments. The parameters were measured at 3 borders because the contours were underwent changes in the proximal fragment (head), gap (neck), and distal fragment (shaft). The radii in the axial plane were measured at the head and neck positions. The measurements of the radii showed that the radii increased with the increase in the flatness of the surface and the radii decreased with the increase in the surface curvature. The radii of the head in axial plane were measured at 3 positions (Head_Top: top of the implant head part, Head_Mid: middle of the implant head part, and Head_Bot: bottom of the implant head part) and those of the radii of the neck, at 2 positions (Neck_Top: top of the implant neck part and Neck_Bot: bottom of the implant neck part). The distance between each position was $3 \mathrm{~mm}$ (Fig. 4a). The radii of the coronal plane were measured at 2 positions. First position was measured at the middle of the head center in coronal plane (Head_Mid) and second position was measured at the middle of the shaft center in coronal plane (Shaft_Mid) (Fig. 4b). Two angles were measured at block insertion in the coronal plane: $\theta-1$ was the angle between the contour line of the proximal fragment and the line connecting the end point of the proximal fragment and the end point of the distal fragment, and $\theta-2$ was the angle between the contour line of the distal fragment and the line connecting the end point of the proximal fragment and the end point of the distal fragment. Horizontal distance (Distance X) was measured between the end point of the proximal fragment and the end point of the distal fragment (Fig. 4c). These data were also compared between uni-planar and biplanar osteotomy groups. The correction for the posterior opening gap that was measured at the posterior opening gap ranged from 5 to $16 \mathrm{~mm}$. Therefore, measurements

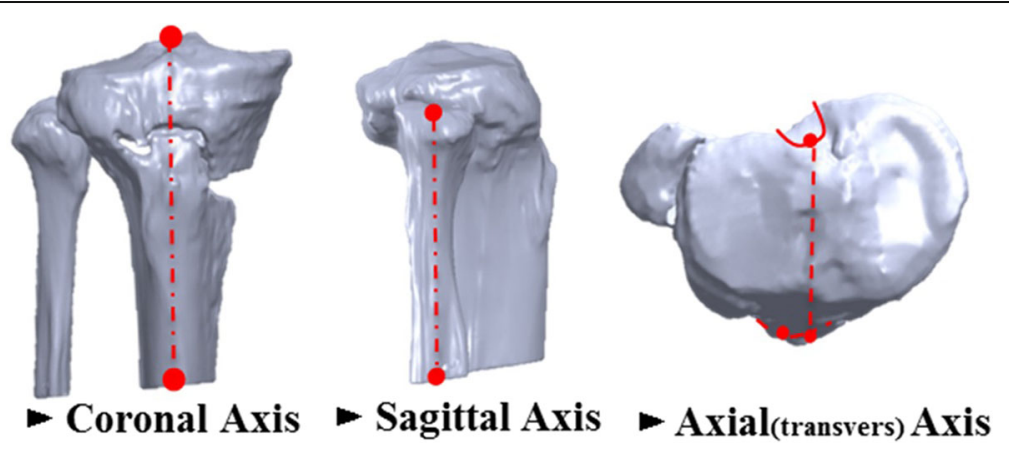

Fig. 3 Bone model re-alignment process 


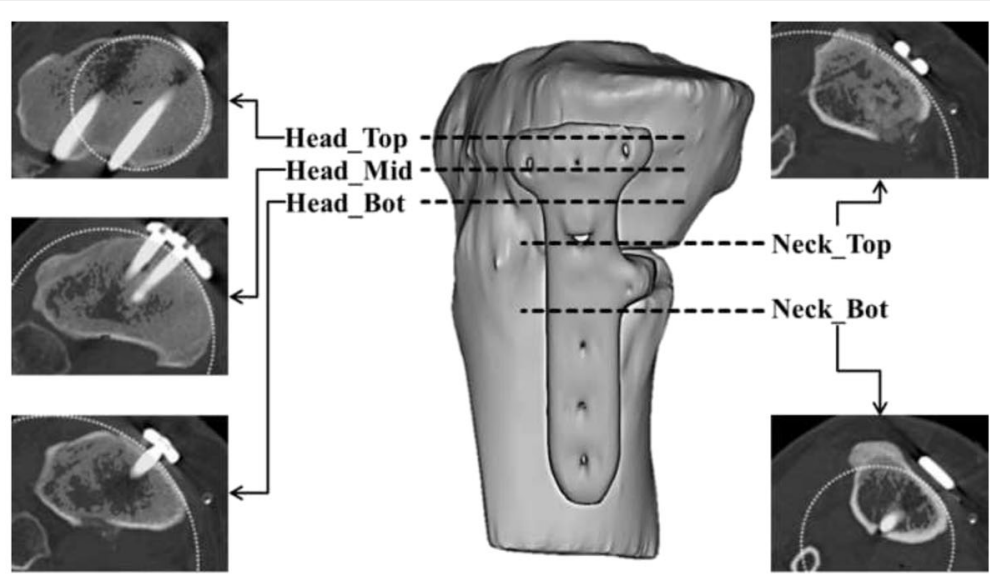

(A)

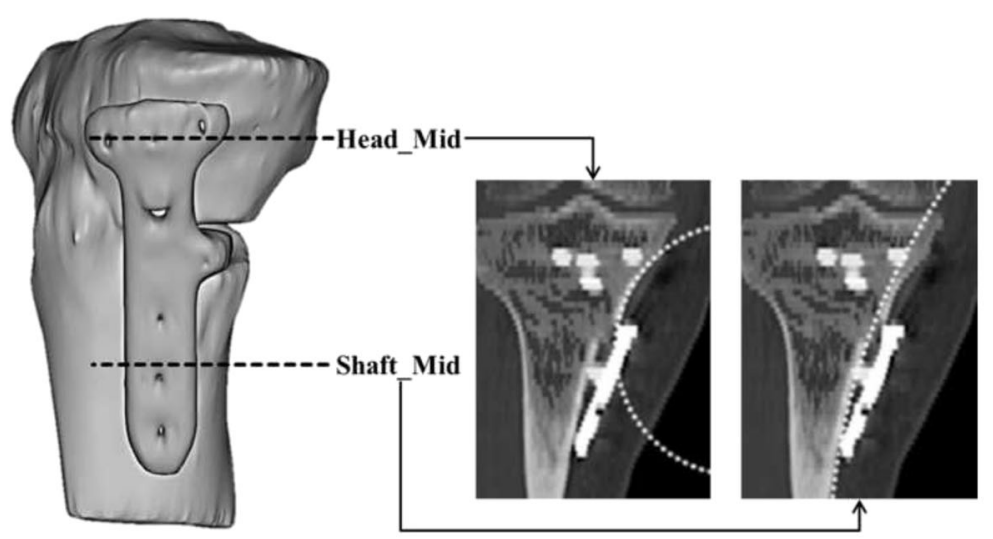

(B)
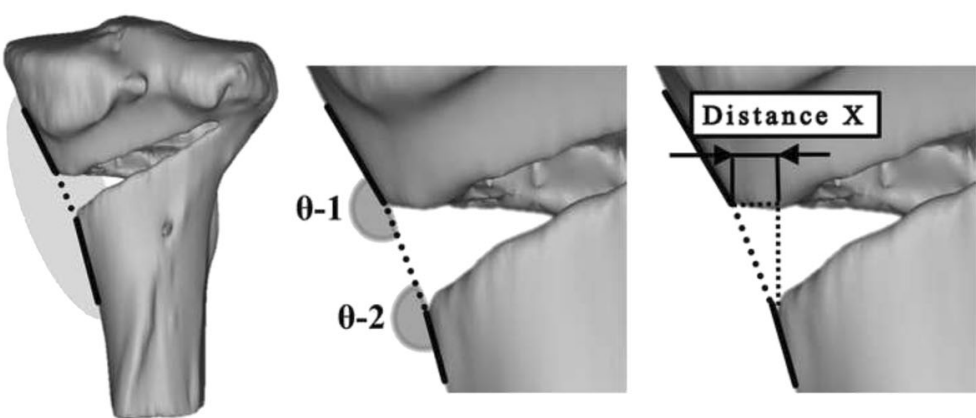

(C)

Fig. 4 Criteria of morphologic factors in the axial plane $\mathbf{a}$ in the coronal plane $\mathbf{b}$ and the angle and horizontal distance $\mathbf{c}$

were divided into 3 groups (Group1: 5-8 $\mathrm{mm}, n=19$, Group2: 9-12 mm, $n=29$, Group3: $13-16 \mathrm{~mm}, n=21$ ) according to the correction amount of the posterior opening gap. Two specialist of this field measured twice with 2-3 weeks interval. The reliability of the measurements was assessed by examining the intrarater and inter-rater reliability using the intra-class correlation coefficient (ICC).

\section{Statistics}

Statistical analyses were performed using the SPSS statistical software v.22 (IBM, New York, USA), in which a significance threshold of 0.05 was set for all statistical comparisons. The Analysis of Variance (ANOVA) test was conducted to verify the change depending on the correction amount of the posterior opening gap. The values obtained for the uni- and bi-planar osteotomy 
were compared by the independent $t$-test. Sample size was calculated from following values ( $\alpha$ error 0.05, power 0.85 , effect size 0.5 : sample size 31 ).

\section{Results}

The inter- and intra-observer reliabilities were satisfactory and the mean values were 0.79 (ranging from 0.68 to 0.88 ) and 0.76 (ranging from 0.69 to 0.84 ), respectively. With regard to the curvature in the axial plane, the radii of the tibial cross-sectional contour at the Head_Top, Head_Mid, and Head_Bot were $61.5 \pm 11.7$, $92.9 \pm 42.1$, and $116.1 \pm 61.3 \mathrm{~mm}$, respectively; there values tended to increase toward the distal direction. The radii of the tibial cross-sectional contour at the Neck_Top, and Neck_Bot were $73.9 \pm 46.3$ and $58.8 \pm$ $32.4 \mathrm{~mm}$, respectively; there values tended to decrease toward the distal direction (Fig. 5a). On the curvature in the coronal plane, the radii of the tibial cross-sectional contour at the Head_Mid and Shaft_Mid were $74.5 \pm$ $18.8,127.0 \pm 27.6 \mathrm{~mm}$, respectively; these values tended to increase toward the distal direction (Fig. 5b). The angles of $\theta-1$ and $\theta-2$ were $167.5 \pm 9.4$ and $165.1 \pm$ $11.0^{\circ}$, respectively, and the Distance $X$ was $6.9 \pm 2.0 \mathrm{~mm}$ (Fig. 5c). There was no significant relationship between the $\theta$ and Distance $\mathrm{X}(p=$ n.s.).

On comparing the values obtained for the uni-planar and bi-planar osteotomy groups, the radii of the Neck_Bot of the bi-planar group were significantly larger than that of the uni-planar group $(p=0.003)$ (Fig. 6a). Osteotomy was performed more distally in the bi-planar group than in the uni-planar group without statistical significance (Fig. 6b). Shaft_Mid and Distance X of GroupI (110.08 $\mathrm{mm}$ and $6.11 \mathrm{~mm}$, respectively) which had lower correction angle were lower than those of GroupII (130.05 $\mathrm{mm}$ and $6.41 \mathrm{~mm}$, respectively) and those of GroupIII (136.35 mm, $8.01 \mathrm{~mm}$, respectively) in coronal plane. There were significant differences $(p=0.023<0.05$ and $p=0.009<0.01$, respectively). In axial plane, there was no significant difference among GroupI, GroupII, and GroupIII (Table 1).

\section{Discussion}

The principal findings of this study were (1) the radii of the tibial cross-sectional contour at the head portion

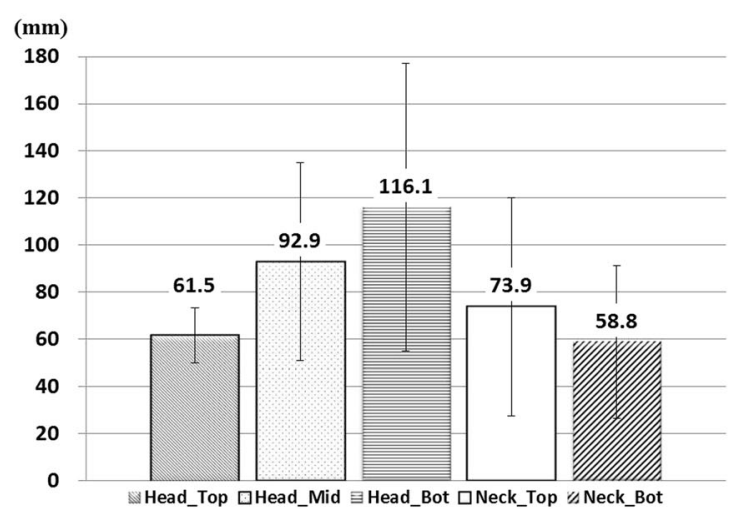

(A)

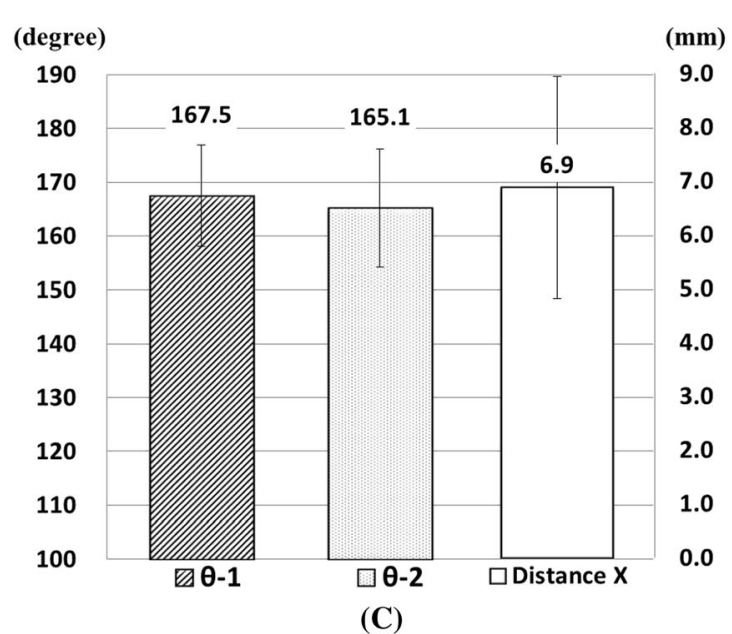

(C)

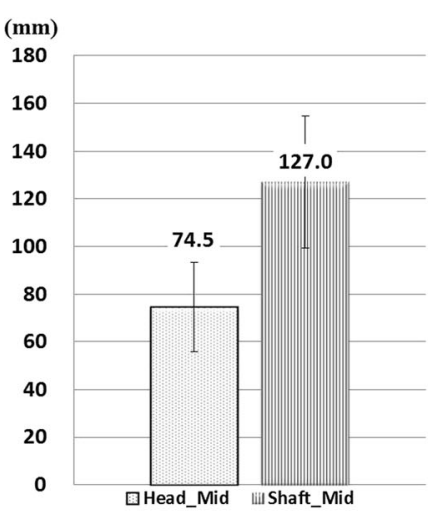

(B)

Fig. 5 Quantitative measurement value of the radii in the axial $\mathbf{a}$ coronal plane $\mathbf{b}$ and the angle and horizontal distance of the gap $\mathbf{c}$ 


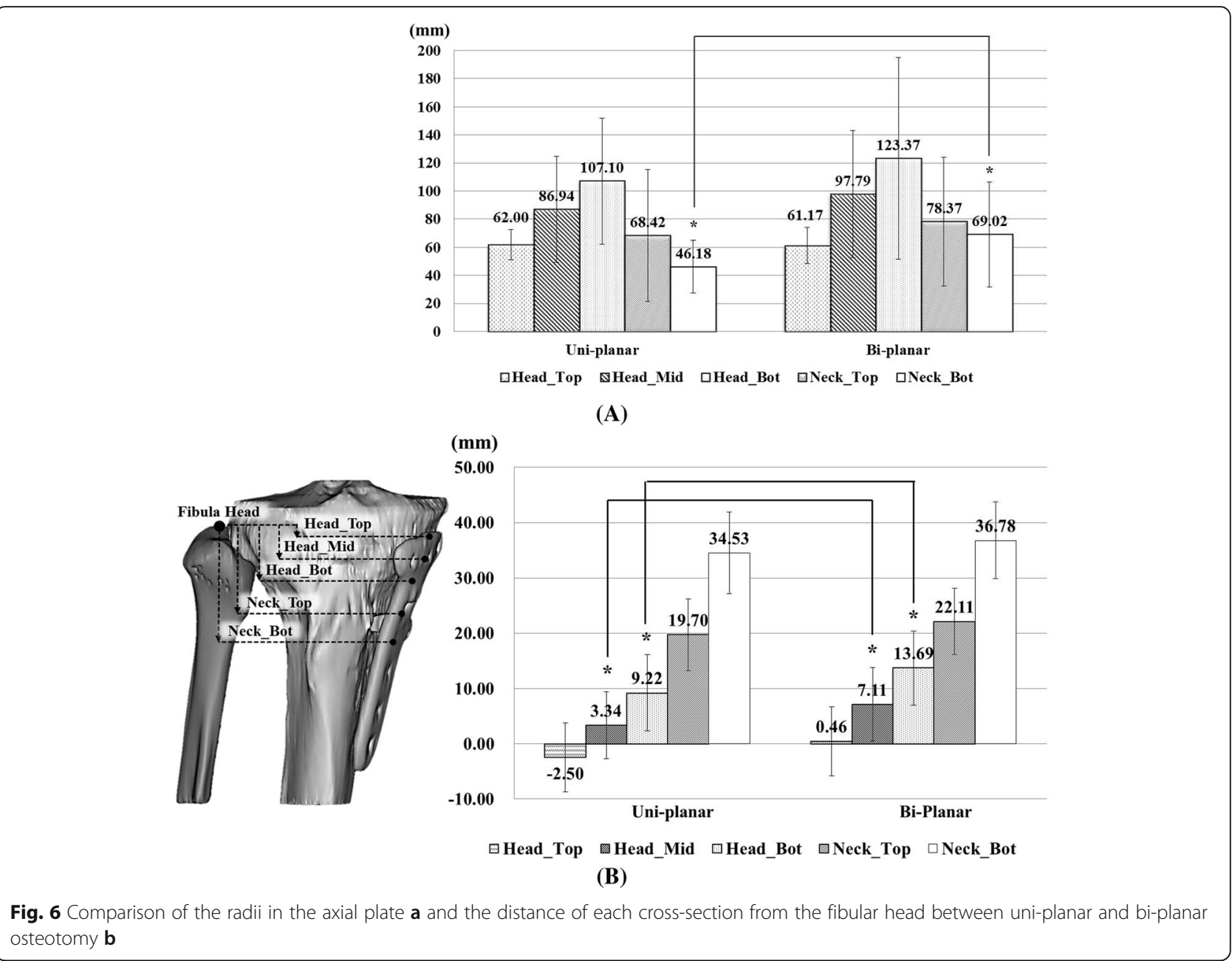

Table 1 Parameters for each correction degree

\begin{tabular}{|c|c|c|c|c|c|c|c|c|}
\hline & & \multicolumn{2}{|c|}{ Group I (5-8 mm, $n=19)$} & \multicolumn{2}{|c|}{ Group II (9-12 mm, $n=29)$} & \multicolumn{2}{|c|}{ Group III (13-16 mm, $n=21)$} & \multirow[t]{2}{*}{$p$-value } \\
\hline & & Mean & SD & Mean & SD & Mean & SD & \\
\hline & Head_Top & $55.8 \mathrm{~mm}$ & 9 & $64.3 \mathrm{~mm}$ & 11.8 & $63.4 \mathrm{~mm}$ & 12.6 & 0.06 \\
\hline & Head_Mid & $84.6 \mathrm{~mm}$ & 37.1 & $103.1 \mathrm{~mm}$ & 49.2 & $86.3 \mathrm{~mm}$ & 33.9 & 0.23 \\
\hline \multirow[t]{5}{*}{ Axial } & Head_Bot & $134.5 \mathrm{~mm}$ & 89.1 & $115.7 \mathrm{~mm}$ & 44 & $99.9 \mathrm{~mm}$ & 32.6 & 0.21 \\
\hline & Neck_Top & $70.8 \mathrm{~mm}$ & 52.5 & 81.4 mm & 50.7 & $66.4 \mathrm{~mm}$ & 32.6 & 0.51 \\
\hline & Neck_Bot & $61.8 \mathrm{~mm}$ & 37.3 & $64.9 \mathrm{~mm}$ & 34.3 & $47.6 \mathrm{~mm}$ & 21.8 & 0.16 \\
\hline & Head_Mid & $71 \mathrm{~mm}$ & 15.7 & $78.5 \mathrm{~mm}$ & 19.6 & $73 \mathrm{~mm}$ & 21 & 0.58 \\
\hline & Shaft_Mid & $110 \mathrm{~mm}$ & 14.7 & $130.1 \mathrm{~mm}$ & 16.2 & $136.4 \mathrm{~mm}$ & 35.6 & $0.02^{*}$ \\
\hline \multirow[t]{3}{*}{ Coronal } & Distance $X$ & $6.1 \mathrm{~mm}$ & 1.3 & $6.4 \mathrm{~mm}$ & 1.7 & $8 \mathrm{~mm}$ & 2.5 & $0.01^{*}$ \\
\hline & $\theta-1$ & $166.7^{\circ}$ & 10.1 & $167.1^{\circ}$ & 9.6 & $168.7^{\circ}$ & 8.9 & 0.77 \\
\hline & $\theta-2$ & $164.7^{\circ}$ & 7.5 & $166.1^{\circ}$ & 11.5 & $164.2^{\circ}$ & 13 & 0.81 \\
\hline
\end{tabular}

Abbreviation: $S D$ standard deviation 
tended to increase from the proximal to distal direction, (2) the radii of the tibial cross-sectional contour at the neck portion tended to decrease from the proximal to distal direction, (3) the radii of the coronal plane tended to increase from the proximal to distal direction, and (4) the angle between the proximal fragment and the distal one varied with the correction amount of the posterior opening gap. The measurements of the radii showed that the radii increased with the increase in the flatness of the surface and the radii decreased with the increase in the surface curvature. Therefore, there was an abrupt change around the posterior opening gap. In the head portion, the surface became flatter toward the distal direction. However, in the neck portion, the surface became curvier toward the distal direction on the axial plane. On the coronal plane, the surface became flatter toward the distal direction.

These changes would be expressed differently according to the correction degree and operative technique. From our results, in coronal plane, Shaft_Mid was increased in the flatness and Distance $\mathrm{X}$ increased as the correction amounts of the posterior opening gap increased. This could imply that it would be necessary to add an optional plate according to the correction degree. Otherwise, the plate should be bended at the both end of the opening gap in coronal plane. The radii of the uni-planar osteotomy were relatively smaller than those of the bi-planar osteotomy in the axial plane. Generally, the proximal portion has more curvature than the distal portion in the axial plane. Therefore, the level at which osteotomy was performed in the uni-planar group was relatively higher than that in the bi-planar group.

With many anthropometric studies on tibial morphologic showing several viewpoints, quantitative studies on the dimension of tibial curvature have been deficient in determining the proper fitting values for OWHTO [20-23]. It could be said that any plate failing to comply with these values did not fit well on the osteotomized bony surface of the proximal tibia, and the quantitative data of this study could provide an anatomical featured design for the OWHTO [24-26]. Proper fitting of the plate to the bony surface is the main factor in OWHTO for delivering appropriate physiological loading and making the stress path effective. Improper fitting of the plate to the bony surface may result in stress concentration of the screw and could lead to a plate fracture or a surgical failure due to loss of its fixation stability [10, 27-30].

The proximal tibia has a unique 3D anatomy as opposed to the mid or distal tibia because multiple structures change abruptly, including the tibial plateau, posterior intercondylar fossa, and posterior cortex. In addition, the center of the proximal tibia is relatively more posteriorly positioned than that of the shaft [15]. Therefore, there would be a possibility of mismatch with conventional plate positioning as described in the Fig. 1. Several anatomical changes occur additionally after OWHTO, and these changes could differ across patients, depending on various factors. The largest anatomical change is due to the primary aspect of the procedure, i.e. the opening of the wedge in the coronal plane: valgus rotation and inferior translation of the distal fragment are relative to the proximal fragment. However, other changes between the proximal and distal fragment were also noted. These secondary changes are unintended and are possibly the result of the orientation of the osteotomy cut and the hinge point relative to the long axis of the tibia [31]. Therefore, in the morphoanalysis, these changes and the morphologic characteristics around the osteotomy site should be considered simultaneously and analysis was performed with three different portions (head, neck, and shaft) in this study.

However, some limitations needed to be considered. First, only the contour of the surface was considered for the plate design. Therefore, information regarding the position of the screw and stability is lacking. Second, a detailed design of the plate has not been established. However, these data could be vital to the proper design of the OWHTO plate. Third, it is not clear how many options should be present according to the correction degree although the tendency of the change has been analyzed according to the correction degree.

\section{Conclusion}

Current plate design should be modified to the surface geometry of the post-correction for the proper fitting. As the correction degree increases, the plate should be bended at the both end of the opening gap in coronal plane.

\section{Abbreviations}

CT: Computed tomography; DICOM: Digital Image Communication in Medicine; HU: Hounsfield Units; OWHTO: Open wedge high tibial osteotomy; ROI: Region of interest

\section{Acknowledgements}

We thank the wonderful staff at the Corentec for their help during the analysis.

\section{Funding}

This research was supported by Basic Science Research program through National Research Foundation of Korea (NRF) funded by the Ministry of Education (2014R1A1A2053356).

\section{Availability of data and materials}

All the data needed to achieve the conclusion are presented in the paper. The raw data cannot be shared as most patients do not want to share their individual data. Additionally, we are preparing patent.

\section{Authors' contributions}

LYS, LMC, PJH, and SDH designed this paper and performed critical revision of the manuscript. YOS and KJW performed data collection and analysis. All authors have agreed to authorship and order of authorship for this manuscript. We confirm that all authors have the appropriate permissions and rights to the reported data. 


\section{Competing interests}

The authors declare that they have no competing interests.

\section{Consent for publication}

Not applicable.

\section{Ethics approval and consent to participate}

This study was approved under B-1502/286-104 (Seoul National University Bundang Hospital Institutional Review Board), and all patients gave consent

\section{Author details}

${ }^{1}$ Central R\&D Center, Corentec Co Ltd, Cheonan, South Korea. ${ }^{2}$ Department of Biomedical Engineering, Yonsei University, Seoul, Korea. ${ }^{3}$ Department of Orthopaedic Surgery, Seoul National University College of Medicine, Bundang Hospital, 166 Gumi-ro, Bundang-gu, Seongnam-si, Gyeonggi-do 463-707, South Korea. ${ }^{4}$ Department of Orthopaedic Surgery, Seoul National University College of Medicine, Seoul National University Hospital, Bundang, South Korea.

\section{Received: 20 May 2016 Accepted: 29 September 2016}

\section{Published online: 10 October 2016}

\section{References}

1. Amis AA. Biomechanics of high tibial osteotomy. Knee Surg Sports Traumatol Arthrosc. 2012;21(1):197-205.

2. Han SB, Bae JH, Lee SJ, Jung TG, Kim KH, Kwon JH, Nha KW. Biomechanical properties of a new anatomical locking metal block plate for opening wedge high tibial osteotomy: uniplane osteotomy. Knee Surg Relat Res. 2014;26(3):155-61.

3. Kim MK, Ha JK, Lee DW, Nam SW, Kim JG, Lee YS. No correction angle loss with stable plates in open-wedge high tibial osteotomy. Knee Surg Sports Traumatol Arthrosc. 2015;23(7):1999-2006

4. Lee DC, Byun SJ. High tibial osteotomy. Knee Surg Relat Res. 2012:24(2):61-9.

5. Lee SC, Jung KA, Nam CH, Jung SH, Hwang SH. The short-term follow-up results of open wedge high tibial osteotomy with using an Aescula open wedge plate and an allogenic bone graft: the minimum 1-year follow-up results. Clin Orthop Surg. 2010;2(1):47-54.

6. Agneskirchner JD, Freiling D, Hurschler C, Lobenhoffer P. Primary stability of four different implants for opening wedge high tibial osteotomy. Knee Surg Sports Traumatol Arthrosc. 2006;14(3):291-300.

7. Cotic M, Vogt S, Hinterwimmer S, Feucht MJ, Slotta-Huspenina J, Schuster T, Imhoff AB. A matched-pair comparison of two different locking plates for valgus-producing medial open-wedge high tibial osteotomy: peek-carbon composite plate versus titanium plate. Knee Surg Sports Traumatol Arthrosc. 2015;23(7):2032-40.

8. Nelissen EM, van Langelaan EJ, Nelissen RG. Stability of medial opening wedge high tibial osteotomy: a failure analysis. Int Orthop. 2009;34(2):217-23.

9. Schroter S, Gonser CE, Konstantinidis L, Helwig P, Albrecht D. High complication rate after biplanar open wedge high tibial osteotomy stabilized with a new spacer plate (Position HTO plate) without bone substitute. Arthroscopy. 2011;27(5):644-52.

10. Valkering KP, van den Bekerom MP, Kappelhoff FM, Albers GH. Complications after tomofix medial opening wedge high tibial osteotomy. J Knee Surg. 2009;22(3):218-25.

11. Luo CA, Lin SC, Hwa SY, Chen CM, Tseng CS. Biomechanical effects of plate area and locking screw on medial open tibial osteotomy. Comput Methods Biomech Biomed Engin. 2015;18(12):1263-71.

12. Staubli AE, De Simoni C, Babst R, Lobenhoffer P. TomoFix: a new LCPconcept for open wedge osteotomy of the medial proximal tibia-early results in 92 cases. Injury. 2003;34 Suppl 2:B55-62.

13. Lee YS, Jung YB. Posterior cruciate ligament: focus on conflicting issues. Clin Orthop Surg. 2013;5(4):256-62.

14. Lee YS, Won JS, Oh WS, Park HG, Lee BK. Lateral tibial bone mineral density around the level of the proximal tibiofibular joint. Knee Surg Sports Traumatol Arthrosc. 2013;22(7):1678-83.

15. Lee YS, Ra HJ, Ahn JH, Ha JK, Kim JG. Posterior cruciate ligament tibial insertion anatomy and implications for tibial tunnel placement. Arthroscopy. 2011:27(2):182-7.

16. Lee YS, Moon GH. Comparative analysis of osteotomy accuracy between the conventional and devised technique using a protective cutting system in medial open-wedge high tibial osteotomy. J Orthop Sci. 2015;20(1):129-36.
17. Lee YS, Kim MG, Byun HW, Kim SB, Kim JG. Reliability of the imaging software in the preoperative planning of the open-wedge high tibial osteotomy. Knee Surg Sports Traumatol Arthrosc. 2015;23(3):846-51.

18. Raja Izaham RM, Abdul Kadir MR, Abdul Rashid AH, Hossain MG, Kamarul T. Finite element analysis of Puddu and Tomofix plate fixation for open wedge high tibial osteotomy. Injury. 2011;43(6):898-902.

19. Sarmah SS, Patel S, Hossain FS, Haddad FS. The radiological assessment of total and unicompartmental knee replacements. J Bone Joint Surg Br. 2012; 94(10):1321-9.

20. Westrich GH, Laskin RS, Haas SB, Sculco TP. Resection specimen analysis of tibial coverage in total knee arthroplasty. Clin Orthop Relat Res. 1994;309: $163-75$

21. Westrich GH, Haas SB, Insall JN, Frachie A. Resection specimen analysis of proximal tibial anatomy based on 100 total knee arthroplasty specimens. J Arthroplasty. 1995;10(1):47-51.

22. Kwak DS, Surendran S, Pengatteeri YH, Park SE, Choi KN, Gopinathan P, et al Morphometry of the proximal tibia to design the tibial component of total knee arthroplasty for the Korean population. Knee. 2007;14(4):295-300.

23. Uehara K, Kadoya Y, Kobayashi A, Ohashi H, Yamano Y. Anthropometry of the proximal tibia to design a total knee prosthesis for the Japanese population. J Arthroplasty. 2002;17(8):1028-32

24. El-Azab H, Halawa A, Anetzberger $\mathrm{H}$, Imhoff $A B$, Hinterwimmer $\mathrm{S}$. The effect of closed- and open-wedge high tibial osteotomy on tibial slope: a retrospective radiological review of 120 cases. J Bone Joint Surg Br. 2008;90(9):1193-7.

25. Billings A, Scott DF, Camargo MP, Hofmann AA. High tibial osteotomy with a calibrated osteotomy guide, rigid internal fixation, and early motion. Long-term follow-up. J Bone Joint Surg Am. 2000;82(1):70-9.

26. Saito $T$, Kumagai $K$, Akamatsu $Y$, Kobayashi $H$, Kusayama $Y$. Five- to ten-year outcome following medial opening-wedge high tibial osteotomy with rigid plate fixation in combination with an artificial bone substitute. Bone Joint J. 2014:96-B(3):339-44

27. Kolb W, Guhlmann H, Windisch C, Kolb K, Koller H, Grutzner P. Openingwedge high tibial osteotomy with a locked low-profile plate. J Bone Joint Surg Am. 2009;91(11):2581-8

28. Zaki SH, Rae PJ. High tibial valgus osteotomy using the Tomofix plate-mediumterm results in young patients. Acta Orthop Belg. 2009;75(3):360-7.

29. Brosset T, Pasquier G, Migaud H, Gougeon F. Opening wedge high tibial osteotomy performed without filling the defect but with locking plate fixation (TomoFix) and early weight-bearing: prospective evaluation of bone union, precision and maintenance of correction in 51 cases. Orthop Traumatol Surg Res. 2011;97(7):705-11.

30. Noyes FR, Mayfield W, Barber-Westin SD, Albright JC, Heckmann TP. Opening wedge high tibial osteotomy: an operative technique and rehabilitation program to decrease complications and promote early union and function. Am J Sports Med. 2006;34(8):1262-73.

31. Teeter MG, Leitch KM, Pape D, Yuan X, Birmingham TB, Giffin JR. Radiostereometric analysis of early anatomical changes following media opening wedge high tibial osteotomy. Knee. 2015;22(1):41-6.

\section{Submit your next manuscript to BioMed Central and we will help you at every step:}

- We accept pre-submission inquiries

- Our selector tool helps you to find the most relevant journal

- We provide round the clock customer support

- Convenient online submission

- Thorough peer review

- Inclusion in PubMed and all major indexing services

- Maximum visibility for your research

Submit your manuscript at www.biomedcentral.com/submit
Biomed Central 